\title{
Effects of Incubation Period and Fasting Time on the Duodenum Morphology from One- Day-Old Commercial Broiler Chicks: An Histomorphometrical Study
}

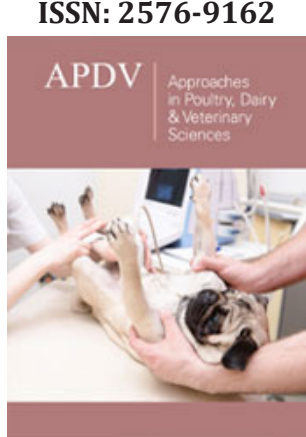

*Corresponding author: Fernanda Radicchi Campos Lobato de Almeida, Department of Morphology, Institute of Biological Sciences, Brazil

Submission: 眥January 21, 2019

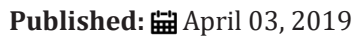

Volume 5 - Issue 5

How to cite this article: Marina A A, Thaís GS, Egladison JC, Hélio C-G, Fernanda RCL d A. Effects of Incubation Period and Fasting Time on the Duodenum Morphology from One-Day-Old Commercial Broiler Chicks: A Histomorphometrical Study. Appro Poult Dairy \& Vet Sci 5(5). APDV.000625.2019. DOI: 10.31031/APDV.2019.05.000625

Copyright@ Fernanda Radicchi Campos Lobato de Almeida, This article is distributed under the terms of the Creative Commons Attribution 4.0 International License, which permits unrestricted use and redistribution provided that the original author and source are credited.

\author{
Marina Antunes Augusto ${ }^{1}$, Thaís Garcia Santos ${ }^{1}$, Egladison João Campos ${ }^{2}$, \\ Hélio Chiarini-Garcia ${ }^{1}$ and Fernanda Radicchi Campos Lobato de Almeida ${ }^{1 *}$ \\ ${ }^{1}$ Department of Morphology, Brazil \\ ${ }^{2}$ School of Veterinary Sciences, Brazil
}

\begin{abstract}
The early management of one-day-old broiler chicks can have a tremendous impact on their subsequent development. Therefore, the establishment of management practices to improve their growth performance is essential, beginning at the hatchery. The objective of this study was to evaluate the morphological changes of the duodenum mucosa due to different incubation periods and fasting time in one-day-old broiler chicks. Twenty-four one-day-old chicks were divided in two groups of 12 animals according to two hatching periods: 490 and 496 hours. Each group was divided into three subgroups, which were subjected to different fasting periods: zero (control), 24 and 48 hours. Four chicks from each subgroup were euthanized at the respective fasting time and samples of the small intestine were collected for histomorphometrical analysis. The results showed that the incubation period affected body and small intestine weights, which were higher at 490-h $(\mathrm{P}<0.05)$. Additionally, 496 hours of incubation promoted severe alterations in the duodenum wall such as Lower Villus Height (VH), Crypt Depth (CD), Mucosal Height $(\mathrm{MH})$, and Smaller Absorptive Area (AA) $(\mathrm{p}<0.01)$. When evaluating the fasting periods, similar changes in the duodenum wall were also observed, and became more evident at the longer fasting period (48 hours). An incubation/fasting period interaction was observed, revealing that longer incubation period reduced the absorptive area regardless of the fasting period duration. Therefore, longer incubation periods and extended fasting have deleterious effects on the development of the small intestine from broiler chicks, which can limit nutrient utilization and their subsequent growth performance.
\end{abstract}

Keywords: Fasting time; Incubation; Poultry; Performance; Duodenum morphology

Abbreviations: MH: Mucosa Height; VH: Villi Height; DV: Villus Diameter; CD: Crypt Depth; HE: Enterocytes Height; AA: Absorptive Area; V/C: Villus Height to Crypt Depth Ratio

\section{Introduction}

The production chain of broiler chickens has an important position in the world poultry industry, especially in Brazil, which is the third world producer but the first in terms of technology [1]. This is mainly due to its fast production cycle, reduced production costs compared to other protein sources, leading to an increase consumption by the massive world population [2]. To meet such a demand, quality of the one-day-old chicks must fulfil all requirements of the production cycle from birth to slaughter to improve production indexes $[3,4]$.

One-day-old-chicks come from hatcheries in which most of them employ multiple stage type incubators. In this system, eggs are incubated at least twice a week until the end of the incubation period, leading to eggs at different stages of embryonic development in the same environment [5]. Hence, eggs hatch at different times, and the hatching range varies from 24 to 48 hours. However, to facilitate management, all chicks are removed from the hatchery at the same time, giving rise to individuals born at different stages of development (19 to 21 days after incubation) [5,6]. Giving this scenario, chicks that are born first remain longer in the birth chambers, exposed to early dehydration. Such a procedure may have a tremendous impact on the chicks' subsequent development mainly due to lack of feeding uniformity, as 
there are chicks that may be subjected to at least 48 hours fast [7]. Thus, the fasting period will reflect on the chicks' intestinal development, because the presence of food in the intestinal lumen is an essential stimulus for growth and maturation [8].

During the first week of life, the chicks develop physiological adaptations in response to the change from an intra- to an extraegg environment and thus switching from an endogenous to an exogenous diet [9]. In this period, the gastrointestinal tract develops up to five times faster than the other organs $[10,11]$, and although anatomically complete, its functional capacity has not come to a complete maturation. In this sense, it is essential that the small intestine rapidly reaches its functional capacity so that the nutrients are used more efficiently [10-12].

One-day-old chicks' uniformity is increasingly important because it contributes to the economic efficiency of the flock, and for this reason, the identification of technical problems is essential to produce excellent quality chicks. The incubation time and the gap between birth and access to first feeding, if inadequate, can interfere in the development of organs, such as the small intestine and consequently, in its capacity to absorb nutrients. Considering that the body weight of one-day-old chicks has a high correlation with body weight at slaughter, inadequate management during this period may reduce the chick's initial viability thus affecting body weight at slaughter, leading to low profitability. Considering these issues, the present study aimed to evaluate the effects of different incubation periods, associated to different fasting times, on the morphology of the duodenum in one-day-old broiler chicks using histomorphometrical approaches.

\section{Materials and Methods}

\section{Ethical committee}

The present study was performed according to the resolution 01/2010 of the Animal Experimentation Control Council. The protocol was approved by the Commission of Ethics in the use of Animals by the Federal University of Minas Gerais (protocol\# 289 / 215).

\section{Animals and histological procedures}

Twenty-four broiler chicks (Cobb breeders) from a commercial hatchery were randomly selected during the normal hatching process, and divided in two groups of 12 chicks each, according to two hatching periods: 490 and 496 hours. All birds were weighed and subsequently allocated to three subgroups, which were subjected to different fasting times: zero (control), 24 and 48 hours. At the end of the respective fasting period, four chicks from each experimental group were euthanized by decapitation and necropsied for the collection of the small intestine, which was weighed, and fragments of the duodenum were taken. Afterwards, these fragments were fixed by immersion in Bouin's solution for 24 hours and embedded in paraffin (Paraplast, Merck). Five $\mu \mathrm{m}$ thickness histological sections were stained with hematoxylin and eosin and used for morphological and histomorphometrical analysis.

\section{Histomorphometrical analysis}

The histomorphometrical studies of the duodenum were performed through the use of digital images obtained from an Olympus BX51 photomicroscope equipped with a Q-Color 3 (Olympus) digital camera. Using the Image-Pro Express program (Media Cybernetics), several measurements were obtained from the digital images of the duodenum wall:

1. Duodenal Mucosa Height (HM)- from the muscularis mucosae up to the apex of the villus;

2. Villi Height (HV), from the base up to the apex of the villus;

3. Villi Diameter (DV);

4. Intestinal Crypt Depth (CD), from the muscularis mucosae up to the base of the villus and

5. height of the enterocytes (HE). All digital images were obtained in an x80 magnification and for each parameter evaluated at least ten measurements were obtained.

In order to determine if the effects of the incubation period and fasting time could functionally affect the intestinal absorption capacity, it was determined the relationship between villi and intestinal crypt as well as the area of intestinal absorption, using the formula described by Kisielensky et al. [13].

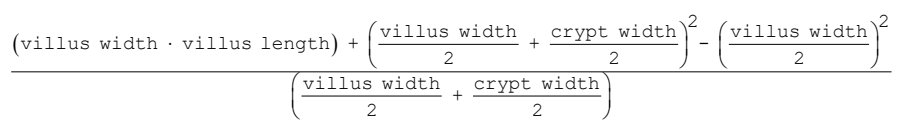

\section{Statistical analysis}

The trial design was a $2 \times 3$ factorial arrangement in a completely randomized design, where the factors included in the model were incubation period (490- and 496-hours), fasting time (0, 24 and 48 hours) and their interactions. Chicks were the experimental unit and all the statistical analyses were conducted using the Software SAS [14]. Significance was established at $\mathrm{p}<0.05$ for all the analyses, and the results are presented as mean \pm standard error (SEM).

\section{Results and Discussion}

\section{Biometrical data}

Table 1 describes the effects of incubation period and fasting time on body and small intestine weights of the broiler chicks. Body weight was affected by the incubation period, as chicks from the 490-h group showed higher body weight compared to their 496h counterparts $(\mathrm{p}<0.05)$. Concomitantly, fasting time also affected body weight, as chicks subjected to 48 hours fasting presented lower body weights compared to the other experimental groups $(p<0.05)$. In addition, small intestine weight decreased with longer incubation and fasting periods $(\mathrm{p}<0.05)$. Interestingly, incubation period did not affect the small intestine to body weight ratio. However, this ratio increased with fasting period, demonstrating a non-proportional relationship between body weight and small intestine weight $(\mathrm{p}<0.05)$. 
Table 1: Body and small intestine weights (g) of broiler chicks submitted to different incubation periods (490 and 496 hours) and fasting times (0, 24 and 48 hours). a,b,c means followed by different superscripts in the same column differ $(\mathrm{p}<0.05) .{ }^{*}$ Interaction between fasting time and incubation period.

\begin{tabular}{|c|c|c|c|}
\hline Treatments & Body Weight (BW, g) & Small Intestine Weight (SIW, g) & SIW/ BW \\
\hline \multicolumn{4}{|c|}{ Incubation Period } \\
\hline $490(n=12)$ & $45.0 \pm 0.7^{\mathrm{a}}$ & $1.55 \pm 0.03^{\mathrm{a}}$ & $0.034 \pm 0.001^{\mathrm{a}}$ \\
\hline $496(n=12)$ & $38.3 \pm 0.7^{b}$ & $1.32 \pm 0.03^{b}$ & $0.034 \pm 0.002^{\mathrm{a}}$ \\
\hline \multicolumn{4}{|c|}{ Fasting Time } \\
\hline $0(n=8)$ & $44.2 \pm 0.9^{\mathrm{a}}$ & $1.20 \pm 0.03^{\mathrm{a}}$ & $0.027 \pm 0.002^{\mathrm{a}}$ \\
\hline $24(n=8)$ & $42.3 \pm 0.9^{\mathrm{a}}$ & $1.73 \pm 0.03^{b}$ & $0.041 \pm 0.001^{\mathrm{b}}$ \\
\hline $48(n=8)$ & $38.3 \pm 0.9^{b}$ & $1.37 \pm 0.03^{c}$ & $0.036 \pm 0.002^{\mathrm{b}}$ \\
\hline \multicolumn{4}{|c|}{ Probability } \\
\hline $\begin{array}{l}\text { Incubation period } \\
\text { (IP) }\end{array}$ & $\mathrm{p}<0.001$ & $\mathrm{p}<0.001$ & $\mathrm{p}<0.001$ \\
\hline Fasting time $(\mathrm{F})$ & $\mathrm{p}<0.05$ & $\mathrm{p}<0.05$ & $\mathrm{p}<0.001$ \\
\hline $\begin{array}{l}\text { FxIP* }(\mathrm{n}=4 / \mathrm{sub}- \\
\text { group) }\end{array}$ & NS & NS & $\mathrm{p}<0.05$ \\
\hline
\end{tabular}

\section{Histomorphometrical analysis}

The histomorphometrical parameters evaluated in the duodenum wall of broiler chicks are shown in Table 2. The 496-hour incubation period affected the duodenum morphology, which was shown by Smaller Villus Height (VH), Crypt Depth (CD), Mucosal Height (HM), and Absorptive Area (AA) (p<0.01). Concomitantly, fasting period also affected histomorphometrical parameters analyzed, which increased along the fasting period $(p<0.01)$ Figure 1.

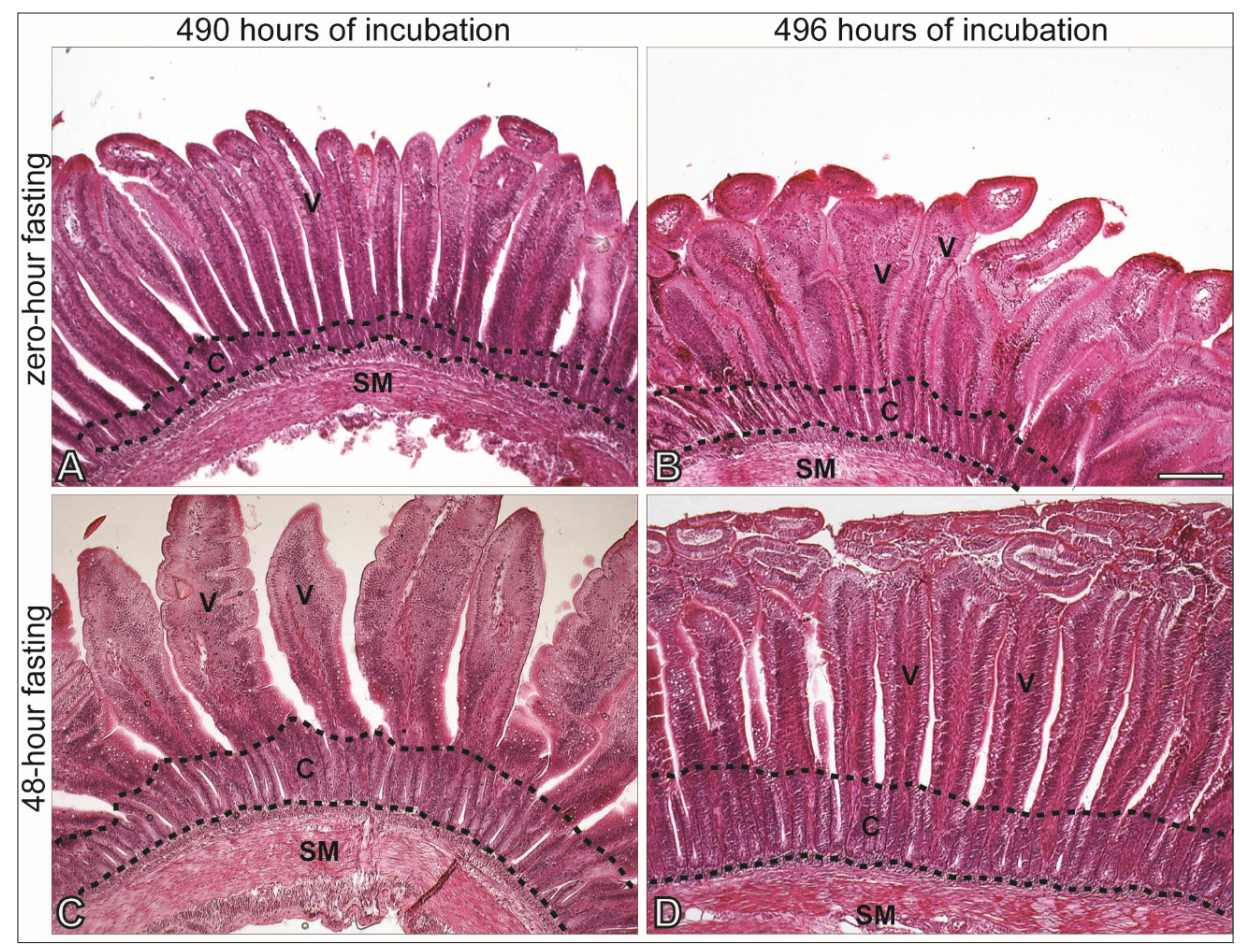

Figure 1: Photomicrograph of the duodenal mucosa showing

A. Interaction between 490 hours of incubation and zero hour fasting

B. Interaction between 496 hours of incubation and zero hour fasting

C. Interaction between 490 hours of incubation and 48 hours fasting and

D. Interaction between 496 hours of incubation and 48 hours fasting. V: Villus; C: Crypt; SM: Submucosa. Bar $=180 \mu \mathrm{m}$. 
Table 2: Histomorphometrical evaluation of broiler chicks submitted to two incubation periods (490 and 496 hours) and three fasting times $(0,24$ and 48 hours). a,b,c means followed by different superscripts within the same column differ $(\mathrm{P}<0.05)$.

\begin{tabular}{|c|c|c|c|c|c|c|c|}
\hline Treatments & VH $(\mu \mathrm{m})$ & $\mathrm{VD}(\mu \mathrm{m})$ & $\mathrm{CD}(\mu \mathrm{m})$ & MH $(\mu \mathrm{m})$ & HE $(\mu \mathrm{m})$ & AA (mm) & $\mathrm{V} / \mathrm{C}$ \\
\hline \multicolumn{8}{|c|}{ Incubation Period } \\
\hline 490 & $587.3 \pm 12.8^{\mathrm{a}}$ & $104.7 \pm 3.3^{\mathrm{a}}$ & $116.2 \pm 4.8^{\mathrm{a}}$ & $704.3 \pm 16.3^{\mathrm{a}}$ & $31.8 \pm 0.8^{\mathrm{a}}$ & $66.5 \pm 2.8^{\mathrm{a}}$ & $5.4 \pm 0.2^{\mathrm{a}}$ \\
\hline 496 & $487.3 \pm 13.5^{\mathrm{b}}$ & $99.2 \pm 3.2^{\mathrm{a}}$ & $91.7 \pm 5.0^{\mathrm{b}}$ & $578.7 \pm 17.2^{\mathrm{b}}$ & $31.3 \pm 0.3^{\mathrm{a}}$ & $53.8 \pm 2.3^{b}$ & $5.5 \pm 0.2^{\mathrm{a}}$ \\
\hline \multicolumn{8}{|c|}{ Fasting Time } \\
\hline 0 & $461.6 \pm 15.7^{\mathrm{b}}$ & $88.6 \pm 4.0^{\mathrm{b}}$ & $71.7 \pm 6.0^{c}$ & $533.0 \pm 20.0^{\mathrm{b}}$ & $27.6 \pm 1.0^{c}$ & $45.3 \pm 2.8^{\mathrm{b}}$ & $6.6 \pm 0.3^{\mathrm{a}}$ \\
\hline 24 & $575.5 \pm 17.0^{\mathrm{a}}$ & $102.8 \pm 4.3^{\mathrm{a}}$ & $102.2 \pm 6.4^{\mathrm{b}}$ & $677.8 \pm 21.6^{\mathrm{a}}$ & $30.8 \pm 0.9^{b}$ & $64.0 \pm 3.0^{\mathrm{a}}$ & $5.7 \pm 0.3^{\mathrm{a}}$ \\
\hline 48 & $574.8 \pm 15.7^{\mathrm{a}}$ & $114.5 \pm 4.0^{\mathrm{a}}$ & $137.8 \pm 6.0^{\mathrm{a}}$ & $713.7 \pm 20.0^{\mathrm{a}}$ & $36.0 \pm 0.9^{a}$ & $71.3 \pm 2.8^{\mathrm{a}}$ & $4.2 \pm 0.3^{\mathrm{b}}$ \\
\hline \multicolumn{8}{|c|}{ Probability } \\
\hline IP & $\mathrm{p}<0.01$ & NS & $\mathrm{p}<0.05$ & $\mathrm{p}<0.01$ & NS & $\mathrm{p}<0.05$ & NS \\
\hline $\mathrm{F}$ & $\mathrm{p}<0.01$ & $\mathrm{p}<0.01$ & $\mathrm{p}<0.01$ & $\mathrm{p}<0.01$ & $\mathrm{p}<0.01$ & $\mathrm{p}<0.01$ & $\mathrm{p}<0.01$ \\
\hline FxIP ${ }^{*}$ & NS & NS & NS & NS & NS & $\mathrm{p}<0.05$ & NS \\
\hline
\end{tabular}

IP: Incubation Period; SE: Stander Error; F: Fasting; NS: Not Significant. *Interaction between fasting time and incubation period.

The interaction between incubation period and fasting time revealed a difference in the duodenal absorptive area in one-dayold chicks $(p<0.05)$ Table 3. Under 490 hours of incubation, the absorptive area remained similar in all groups, regardless of fasting

time. Moreover, there was a reduction of the duodenal absorptive area in chicks subjected simultaneously to 496 hours incubation and from zero to 24 hours fasting, however this difference disappeared in the period of 48 hours fasting.

Table 3: Interaction between fasting times (0, 24, 48 hours) and incubation periods (490 and 496 hours) on absorptive area $(\mathrm{mm})$. A,B,C means followed by different superscripts within the same column differ $(\mathrm{P}<0.05)$. a,b,c means followed by different superscripts within the same row differ $(\mathrm{P}<0.05)$.

\begin{tabular}{|c|c|c|}
\hline Fasting (h) & \multicolumn{2}{|c|}{ Incubation Period (h) } \\
\hline & 490 & 496 \\
\hline 0 & $56.4 \pm 4.0^{\mathrm{Aa}}$ & $34.2 \pm 4.0^{\mathrm{Ab}}$ \\
\hline 24 & $72.0 \pm 4.0^{\mathrm{Aa}}$ & $55.8 \pm 4.6^{\mathrm{Bb}}$ \\
\hline 48 & $71.0 \pm 4.0^{\mathrm{Aa}}$ & $71.6 \pm 4.0^{\mathrm{Ba}}$ \\
\hline
\end{tabular}

The results of this study have generally described that the adaptive response of the intestine and the functions of absorption and digestion are affected by the incubation period and the fasting time to which the animals are subjected. The association between these two variables did not affect the body weight and small intestine of the birds. However, when these data are analyzed separately, the incubation period and the fasting time influenced all parameters analyzed. In accordance to our hypothesis, fasting over 24 hours post-hatching compromises the development of the body and small intestine in one-day-old chicks.

Similar results were previously described, in which live weight reduction was observed in newborn chicks submitted to food restriction in periods that exceeded 24 hours [15-17]. At 48 hours of fasting, there was a $13 \%$ loss of body weight, which seems to be progressive with increased fasting, as observed by other authors. Pinchasov and Noy [18] reported a reduction of $10 \%$ in body weight, however, when the restriction reaches 52 hours, the loss can reach $15 \%$ [19]. Therefore, fasting longer than 24 hours may accentuate body weight loss as observed in this study, since in this period, the yolk reserve is used primarily for the physiological maintenance of the organism $[4,20]$.

Also, in the present study, an increase of 6 hours of incubation significantly influenced the initial performance of the chicks, expressed by the reduction of $15 \%$ of the body weight of the birds when compared to those that were incubated for 490 hours. In commercial poultry, eggs from the same incubator do not hatch synchronously, as variations in temperature, hen's age and egg size may delay shell breaking [21]. However, since newborn chicks are withdrawn simultaneously, those born first are more exposed to high temperatures and deprived of food, and may suffer momentary dehydration and ketosis, resulting in rapid weight loss due to muscle glycogen breakdown as an energy source [8].

It has been observed that periods of prolonged incubation and rapid post-hatching reduce the weight of the small intestine. In agreement with our results, Noy and Sklan [16] also demonstrated drastic reduction of the intestine from newly hatched chicks in 48 hours, since the body weight increased $60 \%$ in that period, against $200 \%$ in normally fed chicks $[15,22]$. 
The duodenal histomorphometric changes reported in the present study provide important information on the relevance of initial management in broiler chicks, since prolonged incubation and fasting time greatly compromised duodenal epithelial morphology. In previous studies, post-hatching food restriction also promoted impairment of the intestinal epithelium, expressed by the reduction of villi associated with crypts, formation of agglomerates of microvilli and reduction of the absorptive area [23-25].

The permanence of intestinal epithelial homeostasis depends on the balance of two cytological phenomena associated with the normal process of cell renewal: proliferation and apoptosis. Duodenal epithelial cells are constantly renewed because of their functional characteristics, which are lost at the apex of the villi and replaced by those that proliferate from stem cells located in the crypts [26]. However, this study demonstrated that prolonged fast may have promoted an imbalance in cellular turnover, leading to structural modification of the epithelium, expressed by the reduction of villi and increase in crypts depth, and consequently lower villi/crypt ratio. Likewise, a longer incubation period negatively impacted all these parameters and also reduced mucosal height. The villous atrophy observed in this study may be caused by increased cell death rate or reduced turnover rate, so that shorter villi can be interpreted in two ways [27]. Our results also reported an association of shortening of villi associated to deep crypt, which may indicate a stimulus in the proliferative activity of stem cells to compensate for cell losses but insufficient, indicating that the rate of apoptosis was higher than the rate of renewal.

The results showed that the longer the fasting time, the lower the villus/crypt ratio. This parameter is considered a good indicator of mucosal renewal, so that short villi with deep crypts seem to indicate lower rate of cell turnover and consequently alteration in absorptive capacity, which may affect subsequent performance. In addition, incubation for 496 hours reduced the extent of the duodenal absorption area, indicating that nutrient absorption is considerably limited.

The results of this study have generally described that the adaptive response of the intestine and the functions of absorption and digestion are affected by the incubation period and the fasting time to which the animals are subjected. These findings suggest that the earlier the chick is fed, the greater the development of the small intestine and duodenal epithelium, resulting in better final digestion and nutrient uptake [16].

\section{Conclusion}

Taken together, our results showed that longer incubation periods and extended fasting time have deleterious effects on the development of the small intestine from broiler chicks, which can limit nutrient utilization and their subsequent growth performance.

\section{Acknowledgement}

The authors are grateful to Rivelli Alimentos for providing the experimental animals and for the technical support for this research.

\section{Conflict of interest}

The authors declare that there is no conflict of interest.

\section{References}

1. Rodrigues ASP, Borges EM, Barwaldt R (2017) A study on the feeding behaviour of broiler chickens using data mining. Scientia Plena 13: 1-7.

2. Singh VP, Pathak V (2017) Quality characterization of giblets of indigenous indian chicken breeds. Int J Curr Microbiol App Sci 6(1): 784-797.

3. Almeida JG, Dahlke F, Maiorka A, Macari M, Furlan RL (2006) Effect of fasting on the interval between birth and housing on performance of broilers from different age matrices. Archives of Veterinary Science 11: 50-54.

4. Camargo JR, Silva IJO, Nazareno AC, Vieira FMC, Castro AC, et al. (2015) Quality of chicks according to the microclimate, waiting time and age of matrices. Brazilian Journal of Agricultural and Environmental Engineering 19: 1079-1085.

5. Tona K, Bamelis F, Ketelaere B, Bruggeman V, Moraes VMB, et al. (2003) Effects of egg storage time on spread of hatch, chick quality, and chick juvenile growth. Poultry Science 82: 736-741.

6. Mather CM, Laughlin KF (1977) Storage of hatching eggs: The effects on early embryonic development. British Poultry Science 18(5): 597-603.

7. Willemsen H, Kamers B, Dahlke F, Han H, Song Z, et al. (2010) Delay in feed access and spread of hatch: importance of early nutrition. Word's Poultry Science Journal 66: 177-188.

8. Maiorka A (2002) Effects of matrix age, fasting, feed energy and glutamine on intestinal development and enzymatic activity of the pancreas chicks. Paulista State University, Brazil.

9. Noy Y, Geyra A, Sklan D (2001) The effect of early feeding on growth and small intestinal development in the posthatch pout. Poultry Science 80: 912-919.

10. Kaminska BZ (1979) Food intake in the Young chick. In: Boorman KN and Freeman BM (Eds.), Food intake regulation in poultry. Poultry Science, Edinburgh, Scotland, pp. 199-206.

11. Calixto LFL (1988) Relationship between body growth and growth of some components of the digestive system of broilers. Dissertation Veterinary School of the Federal University of Minas Gerais, Belo Horizonte, Brazil.

12. Uni Z, Ferket RP (2004) Methods for early nutrition and their potential. Poultry Science 60(1): 101-111.

13. Kisielinski K, Willis S, Prescher A, Klosterhalfen B, Schumpelick V (2002) A simple new method to calculate small intestine absorptive surface in the rat. Clin Exp Med 2(3): 131-135.

14. (2009) SAS-Statistical analysis system: Release 9.1, (software). SAS Institute Inc, Cary, North Carolina, p. 620.

15. Gonzalez E, Kondo N, Saldanha ES, Loddy MM, Careghi C, et al. (2003) Performance and physiological parameters of broiler chickens subjected to fasting on the neonatal period. Poult Sci 82(8): 1250-1256.

16. Noy Y, Sklan D (2000) Hydrolysis and absorption in the small intestines of posthatch chicks. Poult Sci 79(9): 1306-1310.

17. Silva JHV, Ribeiro MLG, Filho JJ, Silva EL (2006) Evaluation of feeding programs for chicks submitted to 72-h post-hatching fasting. Science and Agrotechnology 31: 206-211.

18. Pinchasov Y, Noy Y (1993) Comparison of post-hatch holding time and subsequent early performance of broiler chicks and turkey poults. British Poultry Science 34(1): 111-120.

19. Teixeira ENM, Silva DJHV, Costa FGP, Martins TDD, Givisiez DAF (2009) The effect of post-hatching fasting time, energetic values and inclusion 
of dehydrated egg in pre-initiation and initial diets of broiler chicks. Brazilian Journal of Animal Science 38(2): 314-322.

20. Suzuki T, Noguchi J, Kitamura M, Fujisaki H (2008) Effects of a newly developed post-hatch feed for poultry hatchings on the performance of poultry. The Journal of Poultry Science 45(1): 39-45.

21. Vieira SL, Almeida JG, Lima AR, Conde ORA, Olmos AR (2005) Hatching distribution of eggs varying in weight and breeder age. Brazilian Journal of Poultry Science 7(2): 73-78.

22. Sklan D (2001) Development of the digestive tract of poultry. World's Poultry Science 57(4): 415-428.

23. Awak WA, Ghareeb K, Raheem AS, Bohm J (2008) Effects of dietary inclusion of probiotic and symbiotic on growth performance, organ weights, and intestinal histomorphology of broiler chickens. Poult Sci 88(1): 49-56.
24. Uni Z, Ganot S, Sklan D (1998) Posthatch development of mucosal function in the broiler small intestine. Poult Sci 77(1): 75-82.

25. Olukosi OA, Dono ND (2014) Modification of digesta pH and intestinal morphology with the use of benzoic acid or phytobiotics and the effects on broiler chicken growth performance and energy and nutrient utilization. J Anim Sci 92(9): 3945-3953.

26. Ross MH, Kaye GI, Pawlina W (2003) Histology: A Test and Atlas with cell and molecular biology. ( $4^{\text {th }}$ edn), Lippincott Williams \& Wilkins, Baltimore, Maryland, USA, pp. 434-474.

27. Pluske JR (2001) Morphological and functional changes in the small intestine of the newly weaned pig. In: Piva A, et al. (Eds.), Gut environment of pigs. University Press, Nottingham, England, UK, pp.1-27.

For possible submissions Click below: 